\title{
Determination of Net Radiation Balance using Satellite Imagery in Odeda Local Government Area, Ogun State, Nigeria
}

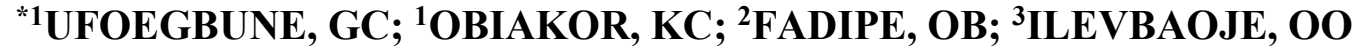

\author{
${ }^{1}$ Federal University of Agriculture, Abeokuta, Nigeria \\ ${ }^{2}$ Federal College of Forestry, Jericho, Ibadan, Nigeria \\ ${ }^{3}$ National Centre for Agricultural Mechanization, Ilorin, Kwara State, Nigeria \\ *Corresponding AuthorEmail: gidufoes2000@yahoo.co.uk,ufoegbunegc@funaab.edu.ng
}

\begin{abstract}
The energy received on the earth surface is responsible for driving activities on the earth and not all energy emitted by the sun reaches the earth surface; some are absorbed, reflected or scattered. There is need to determine the amount of energy that reaches the earth surface since not all the energy emitted from the sun reaches the earth. This energy is also responsible for making our general climate warm which ultimately brings us to the determination of Net radiation. This study applied remote sensing method to determine net radiation over Odeda Local Government Area. A Sentinel image from 2013 covering the study area was downloaded and computations were done using bands 5 and 7 . These bands were used to compute the surface albedo, incoming short wave radiation, incoming long wave radiation and outgoing long wave radiation. The spectral signatures for each component from sentinel data were analysed, using the ArcGIS 10.6. The results showed the net radiation to be of moderate intensity with values ranging between $630.83 \mathrm{wm}^{-2}$ and $684.68 \mathrm{wm}^{-2}$. The mean value over majority of the area was $651.20 \mathrm{wm}^{-2}$ and the albedo of the area was low, with values ranging from 0.0028 to a value of 0.29 which is an indication of low reflectivity. This implied that more radiation was absorbed in the study area which impacted the soil temperature thereby influencing reduced soil moisture and a higher rate of evapotranspiration from plants within the study area.
\end{abstract}

\section{DOI: https://dx.doi.org/10.4314/jasem.v23i9.7}

Copyright: Copyright (C) 2019 Ufoegbune et al. This is an open access article distributed under the Creative Commons Attribution License (CCL), which permits unrestricted use, distribution, and reproduction in any medium, provided the original work is properly cited.

Dates: Received: 07 July 2019; Revised: 15 August 2019; 10 September 2019

Keywords: thermal energy, atmospheric transmissivity, spatial analysis, surface albedo

Solar radiation is one of the thermal energy components of the atmosphere. Radiation is the only form of energy that can travel through the vacuum of outer space (Liang et al., 2010; Bello, 2013). The solar energy which drives nearly all the component of the earth is characterized by short wavelength and wave frequency. There are different laws governing radiation such as the Stefan Boltzmann law, Weins law, Plancks law, among others (Duarte et al., 2006; Bello, 2013). As solar energy impinges on the top of the atmosphere, a sort of energy cascade begins as some of the energy becomes scattered and absorbed. For example, gases like ozone in the atmosphere absorb all the ultraviolet radiation within $0.29 \mu \mathrm{m}$ and water absorbs a lesser amount ranging from $0.9 \mu \mathrm{m}$ to $2.1 \mu \mathrm{m}$. About $20 \%$ of the total incident radiation is absorbed by ozone, water vapour and aerosols. About $30 \%$ of the incident solar radiation is reflected back to space (reflection by cloud tops is $20 \%$, Earth's surface $4 \%$ and then scattering by air and particles $6 \%$ ). Of all these losses, only about $45 \%$ reaches the earth (Bello, 2013). The final interaction of solar radiation with the earth surface is the main focus of this study. The radiant energy incident on the earth's surface can either be absorbed or reflected and usually, only a little amount is absorbed by the surface (this explains why clothes get dried at night or the sudden evaporation of water from surfaces before morning), while the remaining is given off as terrestrial long wave radiation (Bello, 2013). Net radiation can simply be defined as the net amount of radiation fluxes entering and leaving the earth's surface. Net radiation controls sensible and latent heat fluxes and can be referred to as the main energy driving atmospheric movement and planetary layer development. It is the measure of energy available at a given site for evaporation, heating the soil, air and photosynthesis. The radiation components are strongly influenced by surface heterogeneity and by the presence, type and distribution of cloud; and as a result of these, the net surface radiation varies spatially over an environment.

The net radiation has four major components which are; incoming longwave radiation, incoming shortwave radiation, outgoing longwave radiation and outgoing shortwave radiation. Of these four components, the incoming shortwave and long wave components are essentially independent of surface 
condition while the remaining two components strongly depend on the nature of the surface. Net radiation, being a meteorological parameter, can be measured using net radiometer or a long wave or short wave radiometer (Bello, 2013). However, these instruments only take point measurements, that is, they only measure the net radiation of the localty where the instrument is sited and does not aid the understanding of regional net radiation (Samani et al. 2007). Due to the limited number of these instruments in Nigeria especially Abeokuta, obtaining reliable data for net radiation estimates over space and time would be difficult which has made net radiation data in Nigeria rarely available due to the high cost of the instrument and calibration error. This study addressed these questions: What are the alternative methods through net radiation can be determined? Can GIS be a perfect tool for investigating Net Radiation? How possible can the net radiation be obtained in space and time? What are the locations around Odeda local government that experience varying intensities in Net Radiation? This it did by, determining the incoming radiation and outgoing short wave radiation, long wave radiation, albedo and net radiation.

\section{MATERIALS AND METHODS}

Study Area: Odeda is a local government area in Ogun state, Nigeria (figure 1). The local government is located between latitudes $7^{0} 13^{\prime} \mathrm{N}$ and $3^{0} 31^{\prime} \mathrm{N}$ and longitudes $3^{0} 10^{\mathrm{l}} \mathrm{E}$ and $3^{0} 45^{1} \mathrm{E}$. The local government area has an extensive landmass (1263.45 sq km) with Derived Savannah vegetation. The climate is Tropical with double maxima rainfall between April and October having a relatively dry August. The area also has an average temperature of about $27^{\circ} \mathrm{c}$, an annual rainfall of $1238 \mathrm{~mm}$ yearly $(103.2 \mathrm{~mm}$ average rainfall yearly).

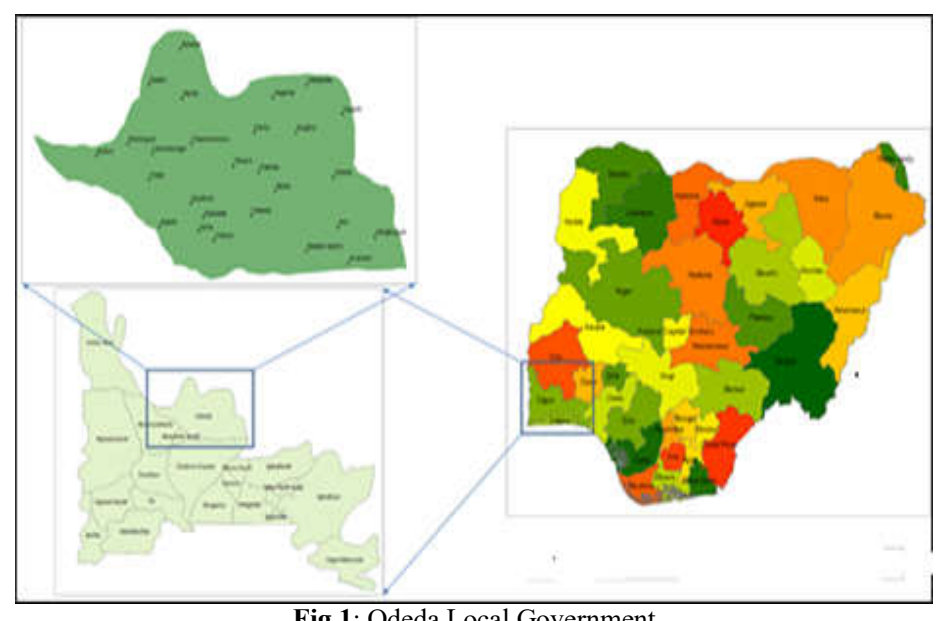

Fig 1: Odeda Local Government

In this study, a Sentinel satellite image data from 2013 was downloaded from earth explorer website (https://earthexplorer.usgs.gov) which was then processed and analysed using the ARCGIS 10.6 software. The analysis followed the guidelines specified by Bastiaanssen (2002). This approach computed net radiation using the Surface Energy Balance Algorithm for Land (SEBAL) model to compute net radiation. This approach computed the incoming longwave and shortwave radiation as well as the outgoing longwave and shortwave radiation alongside the albedo and emissivity.

Computation of the Surface Albedo: $\alpha$ : Surface albedo is a reflection coefficient defined as the ratio of the reflected radiant flux to the incident radiant flux.

$\alpha=\frac{\alpha_{\text {toa }}-\alpha_{\text {path-radiance }}}{\alpha_{\text {sw }^{2}}}$ $\boldsymbol{\alpha}_{\text {toa: }}$ This is the albedo at the top of the atmosphere. This is the albedo unadjusted for atmospheric transmissivity.

$\alpha_{\text {toa }}=\sum\left(\omega_{\lambda} * \rho_{\lambda}\right)$

Where: $\omega_{\lambda}$ is a weighting coefficient for each band computed as follows:

$\omega_{\lambda}=E S U N_{\lambda} / E S U N_{\lambda}$

$\mathrm{ESUN}_{\lambda}$ is the mean solar exo-atmospheric irradiance 2

for each band (W/m / $/ \mu \mathrm{m})$.

$\boldsymbol{\rho}_{\lambda}$ is the reflectivity for each band

$\rho_{\lambda}=\pi \cdot \frac{L_{\lambda}}{E S U N_{\lambda}} \cdot \operatorname{Cos} \theta \cdot d_{r}$ 
Where: $\cos \theta$ is the cosine of the solar incidence angle and it is computed using the header file data on sun elevation angle $(\beta)$ where

$\emptyset=(90-\beta)$

$d_{r}$ is the inverse squared relative earth-sun distance.

$d_{r}=1+0.033(\operatorname{DOY}(2 \pi / 365))$

Where DOY is the sequential day of the year. The angle $($ DOY $\times 2 \pi / 365)$ is in radians. Values for $d_{r}$ range from 0.97 to 1.03 and are dimensionless.

$\mathbf{L}_{\lambda}$ : This is the outgoing radiation energy of the band observed at the top of the atmosphere by the satellite. The units for $\mathrm{L}_{\lambda}$ are $\mathrm{W} / \mathrm{m}^{2} / \mathrm{sr} / \mu \mathrm{m}$.

L_ $\lambda=(($ LMAX-LMIN $) /($ QCALMAXQCALMIN))(DN-QCALMIN)+LMIN .......(7) Where: $\mathrm{DN}$ is the digital number of each pixel

LMAX and LMIN are calibration constants

QCALMAX and QCALMIN are the highest and lowest range of values for rescaled radiance in DN.

$\boldsymbol{\alpha}_{\text {path_radiance: }}$ is the average portion of the incoming solar radiation across all bands that is back-scattered to the satellite before it reaches the earth surface. It ranges between 0.025 and 0.04 and for SEBAL we recommend a value of 0.03 based on Bastiaanssen (2000).

$\boldsymbol{\alpha}_{\text {sw: }}$ is the atmospheric transmissivity which is defined as the fraction of incident radiation that is transmitted by the atmosphere and it represents the effects of absorption and reflection occurring within the atmosphere.

$\tau_{s w}=0.75+2+10^{-5} * Z$

Where $\mathrm{z}$ is the elevation above sea level (m) of the weather station.

Determination of incoming short wave radiation $\left(R_{S \downarrow}\right)$ : $\mathbf{R}_{\mathrm{S} \downarrow}$ : The amount of shortwave radiation that remains available at the surface which is a function of the surface albedo $(\alpha)$.

$R_{s \downarrow}=G_{s c} * \cos \theta * d_{r} * \tau_{s w}$

Where: $\mathrm{G}_{\mathrm{sc}}$ is the solar constant $\left(1367 \mathrm{~W} / \mathrm{m}^{2}\right)$
$\mathbf{R}_{\mathbf{S} \downarrow}:$ is the incoming shortwave radiation

Determination of the incoming long wave radiation: $R_{L \downarrow}$ : is the incoming long wave radiation and it is computed using a modified Stefan-Boltzmann equation with atmospheric transmissivity and a selected surface reference temperature.

$\boldsymbol{R}_{L \downarrow}=\varepsilon_{a} * \sigma * T_{a}^{4}$

Where: $\boldsymbol{\sigma}$ is the Stefan-Boltzmann constant $(5.67 \times 10$

$\mathrm{W} / \mathrm{m} / \mathrm{K}$ ), and $\mathrm{T}_{\mathrm{a}}$ is the near surface air temperature (K).

$\boldsymbol{\varepsilon}_{\mathrm{a}}$ is the atmospheric emissivity (dimensionless)

$\varepsilon_{a}=1.08 *\left(-\ln \tau_{s w}\right)^{265}$

Where: $\boldsymbol{\tau}_{\mathbf{s w}}$ is the atmospheric transmissivity calculated earlier

Therefore, $\boldsymbol{R}_{L \downarrow}=1.08 *\left(-\ln \tau_{\text {sw }}\right)^{.265} * \sigma * T_{\text {cold }}^{4} \cdot$ (12)

$\mathbf{T}_{\mathbf{a}}$ is the near surface air temperature $(\mathrm{K})$.

£: Surface emissivity is the ratio of the thermal energy radiated by the surface to the thermal energy radiated by a blackbody at the same temperature and wavelength and under the same viewing conditions. It is a dimensionless number between 0 (for a perfect reflector and 1(for a perfect emitter).

Determination of outgoing long wave radiation: $R_{L \uparrow}$ : is the outgoing long wave radiation and it is computed using the Stefan-Boltzmann equation with a calculated surface emissivity and surface temperature.

$\mathbf{R}_{\mathrm{L} \uparrow}=\varepsilon_{0} \times \sigma \times \mathbf{T}_{\mathrm{sw}}^{4}$

Where: $\boldsymbol{\varepsilon}_{\mathbf{0}}$ is the "broad-band" surface emissivity (dimensionless)

$\boldsymbol{\sigma}$ is the Stefan-Boltzmann constant $\left(5.67 \times 10^{-8}\right.$ $\mathrm{W} / \mathrm{m} / \mathrm{K}$ )

$\mathbf{T}_{\mathrm{s}}$ is the surface temperature $(\mathrm{K})$.

Determination of Net Radiation: The final step is to then compute the net radiation value over the area: To 
obtain the net radiation $(\mathrm{Rn})$ value from satellite images using SEBAL

$R n=(1-\alpha) R S \downarrow+R L \downarrow-R L \uparrow-(1-$ Eo) $R L \downarrow$ (14)

Where: Rn: Net Radiation; $\alpha$ : albedo of the areas; $\mathrm{RS} \downarrow$ : incoming shortwave radiation; RL $\downarrow$ : incoming long wave radiation; RL个: outgoing long wave radiation; $\varepsilon_{0}$ : broad band surface emissivity (dimensionless)

\section{RESULTS AND DISCUSSIONS}

The results are given in the form of tables and figures. Incoming Shortwave Radiation: The result of the incoming shortwave radiation over the area is given in the figure below. Figure 2 showed the result of the shortwave radiation incidented upon Odeda Local Government Area. The value ranged from $9.06 \mathrm{Wm}^{-2}$ to $263 \mathrm{Wm}^{-2}$. Shortwave radiation, according to Bastiaanssen et al. (2002), ranges from 200$1000 \mathrm{w} / \mathrm{m}^{2}$, to which this study conforms. Areas showing red colour on the map have high shortwave radiation values while areas with yellow colour have moderate shortwave radiation values. Areas with green colours on the map have low shortwave radiation value.

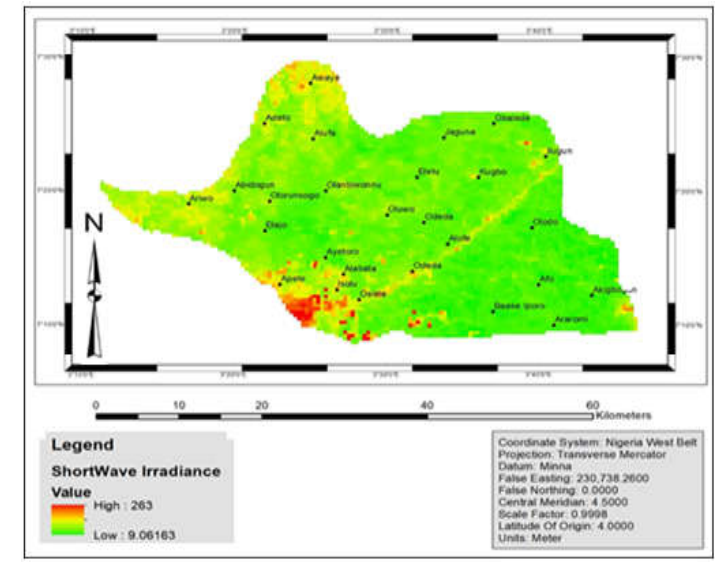

Fig 2: Incoming Shortwave Radiation

The places with red colour revealed places where evaporation as well as evapotranspiration occurred at its highest due to the higher amount of shortwave radiation received. Plants in these areas will thrive well due to exposure to direct sunlight which increases the rate of photosynthesis. The areas with medium values will thrive well but not as much as the areas that received higher values. Likewise for the areas with the low values, plants will grow well but not as well as those which received medium values of shortwave radiation.
Outgoing Shortwave Radiation: The result of the outgoing shortwave radiation is given in the figure 3. Figure 3 showed the result of the spatial computation of the outgoing shortwave radiation within Odeda Local Government Area. It revealed that the outgoing shortwave radiation emitted by the earth's surface ranged from $0.79 \mathrm{Wm}^{-2}$ and $23 \mathrm{Wm}^{-2}$. These values obviously confirms that not all Areas showing red on the image such as the downward part of Apete as well as Isolu and Osiele are areas on the map which experienced high amount of outgoing shortwave radiation while areas with yellow colours such as Ariwo, Awaye, Ilugun etc. had moderate amount of outgoing shortwave radiation. The places with green colour represent areas where the outgoing shortwave radiation amount was lowest. These areas cover a good portion of the map and some of these areas include, Afo, Olodo, Araromi, Baase Iporo etc.

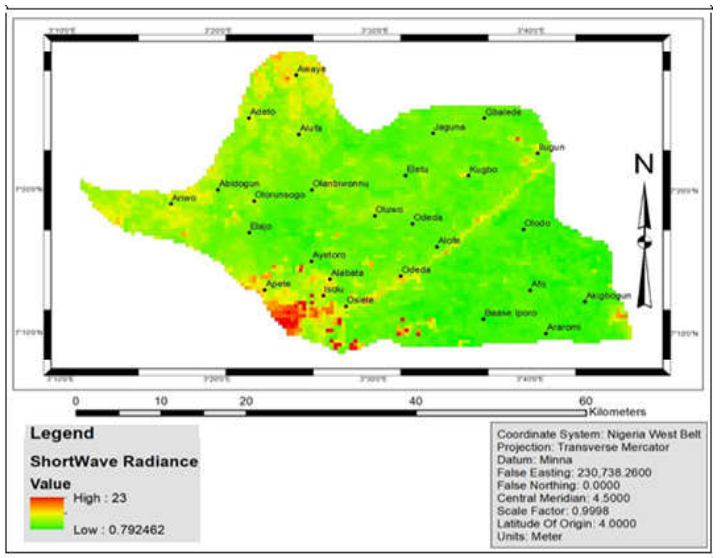

Fig 3: Outgoing shortwave radiation

Albedo of the Study Area: The albedo of the study area is given in the figure 4 . The surface reflectivity ranged from a value of 0.0028 to a value of 0.29 . The mean reflectivity of the land cover surface was however 0.071. This implies that on average the land cover surface within Odeda has a reflectivity of $0.71 \%$ indicating very low reflectivity. This is reflected in the map above as the area is predominantly red (low). The albedo in Odeda LGA ranged from 0.0028 to 0.29 . The lower range value of albedo is represented by red color; the middle range of value is represented by yellow while the highest value is represented by green color. A good and sizeable portion of the land received the lower portion of this range as observed from the image. This implied that Albedo along the areas with orange color was found to be 0.003 or a little higher. The yellow portion in the study area on the image was observed to be relatively scanty which implied an Albedo value of 0.15 or a little higher. Also, very small portion of the area received higher Albedo that is less than or equal to 0.29 ; these areas can be observed by 
green color on the image. Areas such as Akingbade, Olude, and Obantoko experience high albedo while areas with moderate albedo include Ikoto-Ajegunle, Agaru, Akintoye; while majority of the areas experience low Albedo, some of those areas include Orisemo, Soderu, Elegunmowo, Alagbada etc.

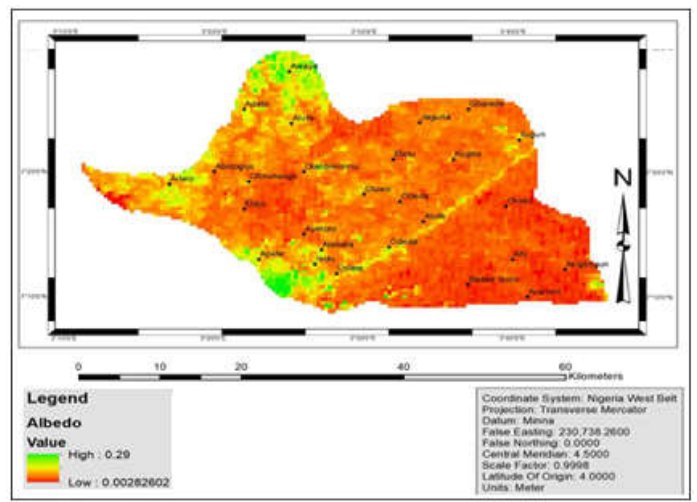

Fig 4: The Surface Albedo of the Area

Incoming Long wave of the Area: The result of the incoming long wave radiation is given in the figure5: The computation of the incoming long wave radiation within Odeda showed that the long wave radiation received by the Earth's surface was ranged between $2.56 \mathrm{wm}^{-2}$ and $263 \mathrm{w} / \mathrm{m}^{2}$. However, the mean shortwave radiance was $64.52 \mathrm{wm}^{-2}$. Areas indicated red shows regions in the local government area experiencing high incoming long wave radiation; some of these areas include Awaye, Elegbada, Olude, Akingbade and Obantoko. Areas indicated yellow are areas with moderate incoming long wave radiation and some of these areas include Oguntoke, Okendi, Ajana etc. while areas indicated green in the image are areas with low incoming long wave radiation some of these areas include Asawo, Balekan, Jaguna etc

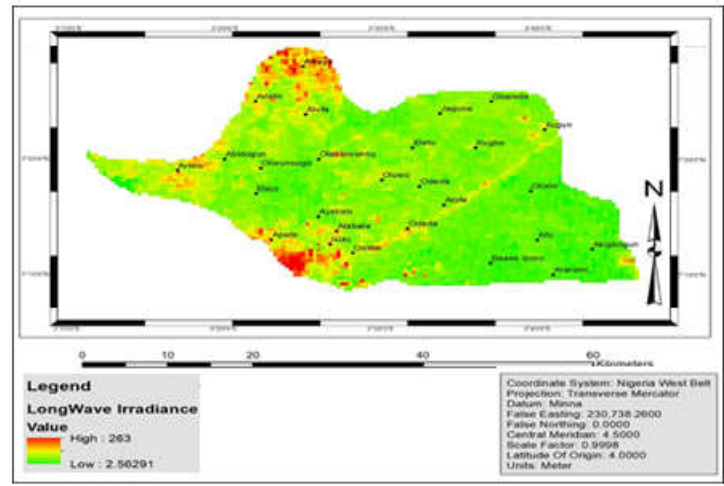

Fig 5: Incoming Long Wave Radiation

Outgoing Long wave Radiation: The outgoing shortwave radiation of Odeda Local government area is given in figure 6 below. The computation of the outgoing long wave radiation within Odeda (figure 6) showed that the long wave radiation emitted by the Earth's surface within the study area was ranged between $0.22 \mathrm{wm}^{-2}$ and $23 \mathrm{wm}^{-2}$. However, the mean long wave radiance was $5.64 \mathrm{wm}^{-2}$. There was more of outgoing long wave radiation in Odeda local government area. This was solely due to the wide spread high surface reflectivity over the area. Areas such as Onisemo, Osawo, Soderu, Bajela, Kemta, Imala etc., other areas in Awaye, kangudu, Olude, Elegbada are areas with high outgoing long wave radiation while some towns in Okendi, Ajana, Okeagbusi, Ariwo, Oguntoke are areas experiencing moderate outgoing levels of long wave radiation, while areas such as Bajela, Soderu, Majo, etc experience low outgoing long wave radiation values.

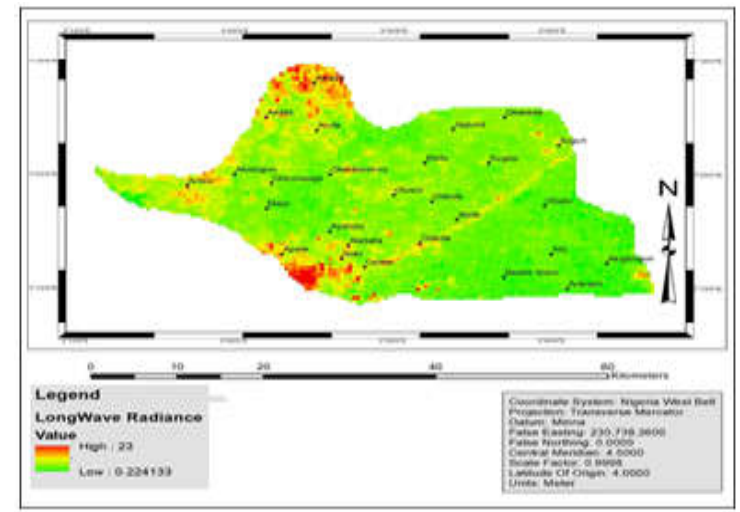

Fig 6: Outgoing Long wave Radiation

Net Radiation Balance of the Study Area: The net radiation of Odeda local government is given in the figure below figure 7 . The net radiation balance of the study area was ranged between $630.83 \mathrm{wm}^{-2}$ and $684.68 \mathrm{wm}^{-2}$. The mean value was however, $651.20 \mathrm{wm}^{-2}$ implying that on average, the net radiation flux was $651.20 \mathrm{wm}^{-2}$.

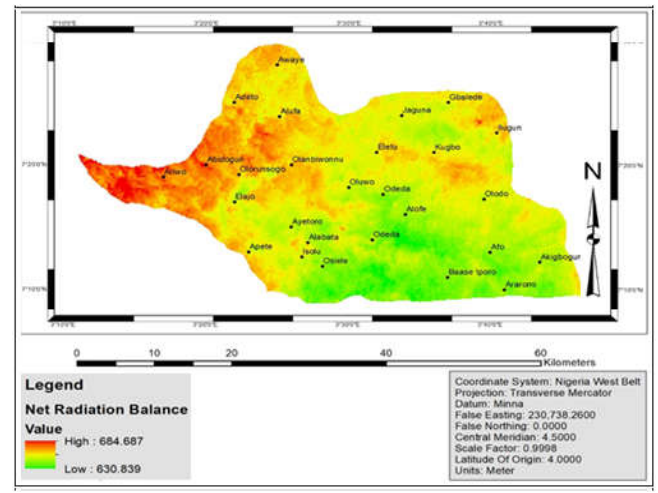

Fig 7: The Net Radiation of Odeda Local Government Area

The net radiation is the energy which is responsible for making the earth habitable for man, animals and also plants; it's the energy that drives the activities of the 
earth. Net radiation plays so many roles in agriculture as well as water resources management part of the roles it plays in agriculture include the provision of necessary energy for all the phenomena concerning biomass production, it also provides energy for the physical processes taking place in plants, soil and also conditions the distribution of temperature and hence crop distribution. Some of the areas of Odeda local government which experience high net radiation include Oja-Ituko, Oguntoke, Ajitadun , Onisapala, Ariwo, Adelakun and this areas would likely have intense Agricultural practices ongoing there increased precipitation, evaporation and very high consumptive water use will be experienced in these areas. Some areas experiencing moderate radiation include Olokemeji, Olowa, Jaguna which implies that the net radiation experienced in these areas will be at moderate level. Areas such as Sawemimo, Kere, Mosafejo are areas with low net radiation values; all these areas are all suitable for agricultural practices, but some areas experience more activities of the component of net radiation than other areas. The values of $R_{n}$ (net radiation) according to (Bastiaanssen et al 2002) range from $\left(100-700 \mathrm{~W} / \mathrm{m}^{2}\right)$ which is in conformity with the observed value of net radiation obtained from the analysis.

Conclusion: The net radiation balance of the area was fairly stable over the study period, practices that could increase that could lead to increase in the albedo of the area should be checked, farmers should also adopt improved cultural practices which would decrease the albedo of soil during cultivation. Evaporation from water bodies could also be reduced by adopting water conservation practices such as the use of films on lakes, reduction in the surface area which will help in the prevention of water escape into nearby residential areas and increase full and adequate river flow if irrigation practices wish to be adopted; while soil conservation practices like mulching and planting of cover crops and a little bit of forestry can be used for soil water conservation.
The allocation of water resources for various purposes can also be obtained, by evaluating the evapotranspiration rate of the area; once the sensible and latent heat of the region is available. This method should be widely adopted not only for climatic studies but also meteorological and environmental studies; but high technical expertise are needed including sufficient satellite data which cannot only be used for present estimation but also for future determination of net radiation balance.

\section{REFERENCES}

Bello N., 2013." Introduction to climatology and biogeography", ISBN: 978-078-813-1 Pp.3244.

Bastiaanssen, W., Menenti, M., Feddes, R; Holtslag, A (1998). A remote sensing Surface Energy Balance Algorithm for Land (SEBAL) - 1 . Formulation. J. Hydrology, 213. 198-212

Bastiannssen, W., Allen, R., Masahiro, T., Trezza, R; Ralf, W. (2002). Advanced Training and User's Manual. Department of Water Resources, University of Idaho. Version 1.0, 1-98.

Duarte H, Dias N, Maggiotto S (2006). Assessing daytime downward long-wave radiation estimates for clear and cloudy skies in Southern Brazil. Agric For Meteorol 139: 171-181

Liang, S., Wang, K., Zhang, X., and Wild, M., 2010. "Review on estimation of land surface radiation and energy budgets from ground measurement, remote sensing and model simulations". IEEE Journal of Selected Topics in Applied Earth Observations and Remote Sensing, 3,pp. 225240.

Samani, Z., Bawazir, S., Bleiwiss, M., Skaggs, R; Tran, V. (2007). Estimating Daily Net Radiation over Vegetation Canopy through Remote Sensing and Climatic Data. J. Irrigate. Drainage Engineer. 133(4), 291-297. 